\title{
Die Temperaturabhängigkeit der Wärmeleitfähigkeiten von gasförmigem Normal- und Ortho-Deuterium bei Temperaturen des flüssigen Wasserstoffes
}

\author{
W. Uebelhack, W. Eichenauer, K. Heinzinger und A. Klemm \\ Max-Planck-Institut für Chemie (Otto-Hahn-Institut), Mainz und \\ Eduard-Zintl-Institut der Technischen Hochschule Darmstadt \\ (Z. Naturforschg. 22 a, 1528-1531 [1967] ; eingegangen am 2. Juni 1967)
}

\begin{abstract}
The relative difference of the thermal conductivities of gaseous ortho- and normal-deuterium has been measured at three different temperatures $18,5^{\circ} \mathrm{K}, 19,8^{\circ} \mathrm{K}$ and $21,1{ }^{\circ} \mathrm{K}$. The results are $0,97 \cdot 10^{-3}, 1,08 \cdot 10^{-3}$ and $1,45 \cdot 10^{-3}$ respectively, with an accuracy of $\pm 10 \%$. They are compared with the relative difference of the viscosities for the two deuterium modifications. This comparison leads to a difference in $f_{\mathrm{tr}}$ and $f_{\text {int }}$ for ortho- and normal-deuterium in the Eucken relation.
\end{abstract}

Die relative Wärmeleitfähigkeitsdifferenz von leichtem Normal- und Para-Wasserstoff bei $20,4^{\circ} \mathrm{K}^{1}$ stimmt innerhalb der Fehlergrenzen mit der von BECKer und StehL ${ }^{2}$ gemessenen relativen Zähigkeitsdifferenz überein. Diese Übereinstimmung ist auf Grund der Euckenschen Beziehung ${ }^{3}$ zu erwarten. Sie lautet für Wasserstoff bei hinreichend tiefen Temperaturen $^{4} M \lambda / \eta=3,75 R+C_{\text {rot }}$. Dabei ist $\lambda$ die Wärmeleitfähigkeit, $\eta$ die Zähigkeit, $M$ die Molmasse, $R$ die molare Gaskonstante und $C_{\text {rot }}$ die Molwärme der Rotation. Für leichten Wasserstoff bei $20,4{ }^{\circ} \mathrm{K}$ befinden sich beide Modifikationen im Grundzustand der Rotation, d. h. $C_{\text {rot }}=0 . \lambda$ ist also direkt proportional $\eta$, und daraus folgt die Gleichheit der relativen Differenzen.

Für Deuterium bei dieser Temperatur gilt das wegen des größeren Trägheitsmomentes nicht mehr. Zwar befinden sich praktisch alle Para-DeuteriumMoleküle im Rotationsgrundzustand, von den OrthoMolekülen ist aber schon ein nicht mehr vernachlässigbarer Teil im ersten angeregten Rotationszustand $(l=2) . C_{\text {rot }}\left(o-\mathrm{D}_{2}\right)$ ist also größer als Null. Entsprechend diesen Erwartungen stimmt auch die von Heinzinger, Eichenauer und Klemm ${ }^{5}$ bei der Temperatur des flüssigen Wasserstoffs gemessene relative Wärmeleitfähigkeitsdifferenz nicht mit der von Becker, Misenta und Stehl ${ }^{6}$ gemessenen relativen Zähigkeitsdifferenz der Deuteriummodifikationen überein. Aber der gefundene Unterschied ist wesentlich größer als man nach der Eucken-Beziehung

1 K. Heinzinger, Z. Naturforschg. 15 a, 1022 [1960]; K. Heinzinger, A. Klemm u. L. Waldmann, Z. Naturforschg. 16 a, 1338 [1961].

2 E. W. Becker u. O. Stehl, Z. Phys. 133, 615 [1952].

3 A. Eucken, Z. Phys. 14, 324 [1913]. erwarten sollte. In dieser Arbeit wurde versucht, durch Messung der relativen Wärmeleitfähigkeitsdifferenz bei noch tieferen Temperaturen festzustellen, ob sich bei praktisch verschwindender Rotationswärme eine Übereinstimmung mit den gemessenen relativen Zähigkeitsunterschieden ergibt.

\section{Apparatur und Ausführung der Messungen}

Für die Messung der Wärmeleitfähigkeit wurden zwei in einem Kupferzylinder eingebaute SchleierMAcher-Zellen benutzt. Die für die Versuche verwendete Apparatur war im Prinzip die gleiche wie bei unseren früheren Messungen ${ }^{1}$. Der Analysenteil wurde nicht benötigt, denn es wurde nur Normalgegen Ortho-Deuterium gemessen. Die Temperatureinstellung erfolgte durch ein Wasserstoffbad, bei dem der Druck über dem flüssigen Wasserstoff mit Hilfe eines Quecksilbermanostaten auf den gewünschten Wert eingestellt werden konnte. Die Druckkonstanz während der Messungen war besser als \pm 1 Torr (entsprechend einer Temperaturschwankung von ca. $\pm 6 \cdot 10^{-3}{ }^{\circ} \mathrm{K}$ ). Auch Darstellung und Reinigung des Deuteriums wurden bereits ausführlich beschrieben ${ }^{5}$. Der Reinheitsgrad des Meßgases wurde mit einem Massenspektrometer geprüft. Die Deuteriumkonzentration war stets größer als 99,9\%.

Die Messungen wurden bei 3 verschiedenen Badtemperaturen ausgeführt. Die eingestellten Drücke über dem flüssigen Wasserstoff waren 760, 524 und

${ }^{4}$ E. A. Mason u. L. Monchick, J. Chem. Phys. 36, 1622 [1962].

5 K. Heinzinger, W. Eichenauer u. A. Klemm, Z. Naturforschg. 18 a, 762 [1963].

${ }^{6}$ E. W. Becker, R. Misenta u. O. Stehl, Z. Phys. 136, 457 [1953]. 
330 Torr. Aus der Dampfdruckkurve des Wasserstoffs ergeben sich daraus die Temperaturen $20,4{ }^{\circ} \mathrm{K}$, $19,1^{\circ} \mathrm{K}$ und $17,8^{\circ} \mathrm{K}$. Um eine Kondensation des Meßgases in den Zellen mit Sicherheit auszuschlieBen, wurde die Badtemperatur von $17,8^{\circ} \mathrm{K}$ nicht unterschritten. Bei dieser Temperatur beträgt der Dampfdruck des Deuteriums nur noch etwa 80 Torr, und die Wärmeleitfähigkeitsmessungen wurden im Druckbereich 40 - 50 Torr ausgeführt, weil bei niedrigeren Drücken wegen der größeren freien Weglänge Wandeffekte auftreten. Der Strom durch die Heizdrähte der Zellen betrug $8 \mathrm{~mA}$. Aus dem Temperaturkoeffizienten der Heizdrähte und dem Absolutwiderstand der Drähte unter Meßbedingungen ergab sich eine Übertemperatur der Heizdrähte von $1,4^{\circ}$. Die Ausführung der Messungen und die Auswertung wurden in den früheren Arbeiten bereits ausführlich beschrieben und sollen deshalb hier nicht wiederholt werden.

\section{Meßergebnisse}

In der Tab. 1 sind die Meßergebnisse zusammengestellt. Die Meßwerte für die relativen Wärmeleitfähigkeitsdifferenzen sind Mittelwerte aus der jeweils in der Tabelle angegebenen Zahl von Einzelmessungen. Die Fehlergrenzen sind die mittleren quadratischen Fehler, die sich aus der Streuung der einzelnen Meßwerte ergeben.

Wegen der komplizierten Gestalt der Meßzellen ${ }^{1}$ ist der Temperaturverlauf in den Meßzellen nicht bekannt, und es läßt sich deshalb eine mittlere Temperatur nicht ausrechnen. Die in der Tabelle angegebenen Temperaturen sind die arithmetischen Mittelwerte zwischen Badtemperatur und Heizdrahttemperatur, d. h. Badtemperatur plus $0,7^{\circ}$. Als Tempera- turungenauigkeit muß also $\pm 0,7^{\circ}$ angenommen werden.

Das Ergebnis aus der früheren Messung ${ }^{5} \Delta \lambda / \lambda=$ $(1,7 \pm 0,3) \cdot 10^{-3}$ kann als zusätzlicher Meßwert in die Diskussion mit einbezogen werden; denn obwohl damals die Badtemperatur auch $20,4{ }^{\circ} \mathrm{K}$ betrug, war die Übertemperatur der Heizdrähte $2,5^{\circ}$. Wir haben diesem Meßwert, der Übertemperatur entsprechend, eine mittlere Temperatur von $21,7^{\circ} \mathrm{K}$ zuzuordnen, mit einer größeren Temperaturungenauigkeit von $\pm 1,3^{\circ}$.

\section{Diskussion}

Theoretische Untersuchungen der Transporteigenschaften gasförmiger Wasserstoffmodifikationen bei tiefen Temperaturen liegen bisher nur für den leichten Wasserstoff vor. Nachdem die Rechnungen von Cohen et al. ${ }^{7}$ mit einem Lennard-Jones-Potential keine gute Übereinstimmung mit den Experimenten gebracht hatten, haben Niblett und Tagayanagi ${ }^{8}$ für den leichten Wasserstoff ein nichtsphärisches Wechselwirkungspotential der Moleküle eingeführt. Es ist anzunehmen, daß dies auch für die Beschreibung der Deuteriummodifikationen notwendig ist. Da aber derartige Rechnungen für Deuterium noch ausstehen, beschränken wir uns hier darauf, mit Hilfe der halbempirischen Eucken'schen Beziehung unsere Wärmeleitfähigkeitsmessungen mit den Viskositätsmessungen von Becker, Misenta und StehL ${ }^{6}$ (s. Tab. 1) zu vergleichen.

In allgemeiner Form kann die Eucken'sche Beziehung geschrieben werden:

$$
(\lambda / \eta) M=f_{\mathrm{tr}} C_{v, \text { tr }}+f_{\mathrm{int}} C_{\mathrm{int}} .
$$

Dabei ist $C_{v, \operatorname{tr}}=\frac{3}{2} R$ die Molwärme der Translation bei konstantem Volumen und $C_{\text {int }}$ die Molwärme der

\begin{tabular}{ccccc}
\hline${ }^{\circ} \mathrm{K}$ & 18,5 & 19,8 & 21,1 & 21,7 \\
\hline$\frac{\lambda\left(\mathrm{o}-\mathrm{D}_{2}\right)-\lambda\left(\mathrm{n}-\mathrm{D}_{2}\right)}{\lambda\left(n-\mathrm{D}_{2}\right)} \cdot 10^{3}$ & $0,97 \pm 0,10$ & $1,08 \pm 0,10$ & $1,45 \pm 0,15$ & $1,7 \pm 0,3$ \\
Zahl der Einzelmessungen & 14 & 6 & 21 & 14 \\
$\frac{\eta\left(\mathrm{o}-\mathrm{D}_{2}\right)-\eta\left(\mathrm{n}-\mathrm{D}_{2}\right)}{\eta\left(\mathrm{n}-\mathrm{D}_{2}\right)} \cdot 10^{3}$ & $0,59 \pm 0,06$ & $0,57 \pm 0,06$ & $0,55 \pm 0,06$ & $0,54 \pm 0,05$
\end{tabular}

Tab. 1. Relative Wärmeleitfähigkeitsdifferenzen und relative Zähigkeitsdifferenzen zwischen Ortho- und Normal-Deuterium für verschiedene Temperaturen. Die $\Delta \lambda / \lambda$-Werte bei $18,5^{\circ} \mathrm{K}, 19,8^{\circ} \mathrm{K}$ und $21,1{ }^{\circ} \mathrm{K}$ entstammen dieser Arbeit, der Wert bei $21,7^{\circ} \mathrm{K}$ einer früheren Arbeit ${ }^{5}$. Die $\Delta \eta / \eta$-Werte sind der Arbeit von Becker, Misenta und Stehr ${ }^{6}$ entnommen.

7 E. G. D. Cohen, M. J. Offerhaus, J. M. J. van Leuwen, B. W. Ross u. J. De Boer, Physica 22, 791 [1956].
8 P. D. Niblett u. K. Tagayanagi, Proc. Roy. Soc. London 250 A, 222 [1959]. 
inneren Freiheitsgrade, die bei unseren Versuchsbedingungen gleich $C_{\text {rot }}$, der Molwärme der Rotation, ist. In der Arbeit von Mason und MonchicK ${ }^{4}$ wird gezeigt, daß gaskinetische Rechnungen für mehratomige Moleküle bei Berücksichtigung inelastischer Stöße unter vereinfachten Annahmen zu den Beziehungen $f_{\text {tr }}=2,5$ und $f_{\text {int }}=\varrho D / \eta$ führen $(\varrho$ ist die Dichte, $D$ der Selbstdiffusionskoeffizient und $\eta$ die Zähigkeit). Es wird dabei angenommen, daß bei Stößen immer nur ein Energiequant zwischen Translation und Rotation ausgetauscht wird. Diese Annahme ist für hinreichend tiefe Temperaturen, also sicher im hier vorliegenden Fall, berechtigt. Für Deuterium bei $20,4{ }^{\circ} \mathrm{K}$ gilt im Rahmen der Meßfehler $( \pm 0,15) \varrho D / \eta=1$. Es ergibt sich damit die Euckensche Beziehung in der ursprünglich von EuCKen selbst vorgeschlagenen Form:

$$
(\lambda / \eta) M=3,75 R+C_{\text {rot }} .
$$

Die Euckensche Beziehung in Form von Gl. (2) ist für leichten Wasserstoff bei Temperaturen unterhalb von $100{ }^{\circ} \mathrm{K}$ experimentell bestätigt ${ }^{4}$.

Nach Gl. (2) ist $\lambda / \eta$ für Ortho- und Normal-Deuterium nur wegen der unterschiedlichen Rotationswärmen der beiden Deuteriummodifikationen verschieden. Wir können

$$
C_{\text {rot }}\left(\mathrm{n}-\mathrm{D}_{2}\right)=\frac{2}{3} C_{\text {rot }}\left(\mathrm{o}-\mathrm{D}_{2}\right)
$$

setzen, denn $C_{\text {rot }}\left(\mathrm{p}-\mathrm{D}_{2}\right)$ ist praktisch gleich Null, da bei den Temperaturen des flüssigen Wasserstoffs näherungsweise alle Para-Moleküle im Grundzustand der Rotation sind. Aus (2) und (3) ergibt sich dann unter Berücksichtigung von $C_{\text {rot }}\left(0-D_{2}\right) /$ $(3,75 R) \ll 1$ näherungsweise :

$$
\begin{aligned}
& \frac{\lambda\left(0-D_{2}\right) / \lambda\left(n-D_{2}\right)}{\eta\left(0-D_{2}\right) / \eta\left(n-D_{2}\right)}=1+\frac{1}{3} \frac{C_{\text {rot }}\left(0-D_{2}\right)}{3,75 R} . \\
& \text { Mit } \quad \frac{\lambda\left(0-\mathrm{D}_{2}\right)-\lambda\left(\mathrm{n}-\mathrm{D}_{2}\right)}{\lambda\left(\mathrm{n}-\mathrm{D}_{2}\right)}=\frac{\Delta \lambda}{\lambda} \\
& \text { und } \quad \frac{\eta\left(\mathrm{o}-\mathrm{D}_{2}\right)-\eta\left(\mathrm{n}-\mathrm{D}_{2}\right)}{\eta\left(\mathrm{n}-\mathrm{D}_{2}\right)}=\frac{\Delta \eta}{\eta}
\end{aligned}
$$

folgt wiederum genähert:

$$
\frac{\Delta \lambda}{\lambda}-\frac{\Delta \eta}{\eta}=\frac{1}{3} \frac{C_{\mathrm{rot}}\left(0-\mathrm{D}_{2}\right)}{3,75 R} .
$$

Aus der Zustandssumme $(Q)$ für Ortho-Deuterium ergibt sich gemäß

$$
C_{\text {rot }}=R \frac{\mathrm{d}}{\mathrm{d} T} T^{2} \frac{\mathrm{d} \ln Q_{\mathrm{rot}}}{\mathrm{d} T}
$$

die Beziehung ${ }^{5}$

$$
\frac{\Delta \lambda}{\lambda}-\frac{\Delta \eta}{\eta}=16 \sigma^{2} e^{-6 \sigma} \equiv a(T)
$$

mit $\sigma=\hbar^{2} / 2 \Theta k T=43,77 / T$.
In $A b b .1$ sind, neben den gemessenen $\left(\frac{\Delta \lambda}{2}-\frac{\Delta \eta}{\eta}\right)-$ Werten mit ihren Fehlergrenzen, $a(T)$ und zwei weitere Kurven aufgetragen, die nachfolgend erläutert werden.

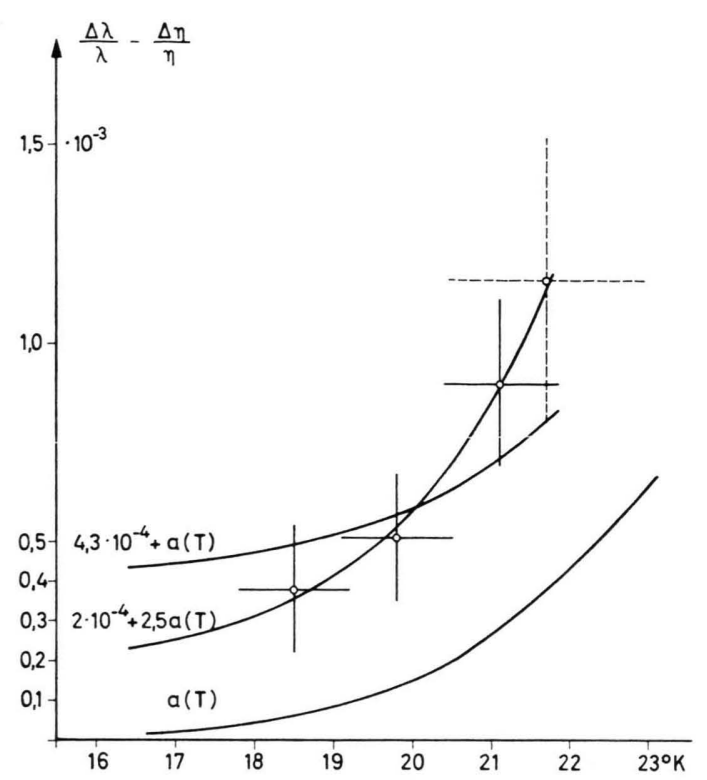

Abb. 1. Experimentelle Werte der Größe

$$
\frac{\lambda\left(0-\mathrm{D}_{2}\right)-\lambda\left(\mathrm{n}-\mathrm{D}_{2}\right)}{\lambda\left(\mathrm{n}-\mathrm{D}_{2}\right)}-\frac{\eta\left(\mathrm{o}-\mathrm{D}_{2}\right)-\eta\left(\mathrm{n}-\mathrm{D}_{2}\right)}{\eta\left(\mathrm{n}-\mathrm{D}_{2}\right)} \text {, sowie }
$$

Kurven unter Verwendung der Funktion $a(T)$ (vgl. Text).

Aus der Abbildung ist ersichtlich, daß Gl. (7) und damit (2) nicht erfüllt ist. Weder verschwindet $\left(\frac{\Delta \lambda}{2}-\frac{\Delta \eta}{\eta}\right)$ bei verschwindender Rotationswärme, noch zeigt die Reihe der Meßpunkte die gleiche Steigung wie die Kurve $a(T)$. Unter Benutzung von $a(T)$ lassen sich die Meßpunkte aber darstellen durch die Beziehung

$$
\frac{\Delta \lambda}{\lambda}-\frac{\Delta \eta}{\eta}=2 \cdot 10^{-4}+2,5 a(T),
$$

die nun zu diskutieren ist.

Bei der Ableitung der Gl. (5) aus der Euckenschen Beziehung (1) wurde angenommen, daß $f_{\mathrm{tr}}\left(\mathrm{o}-\mathrm{D}_{2}\right)=f_{\mathrm{tr}}\left(\mathrm{n}-\mathrm{D}_{2}\right)=2,5$ und $f_{\text {int }}\left(\mathrm{o}-\mathrm{D}_{2}\right)=$ $f_{\text {int }}\left(\mathrm{n}-\mathrm{D}_{2}\right)=1$ sei. Wenn man diese Voraussetzungen fallen läßt, aber $f_{t \mathrm{r}} \approx 2,5$ beibehält, findet man aus (1) genähert:

$$
\begin{aligned}
& \frac{\Delta \lambda}{\lambda}-\frac{\Delta \eta}{\eta}=\frac{f_{\mathrm{tr}}\left(0-\mathrm{D}_{2}\right)-f_{\mathrm{tr}}\left(\mathrm{n}-\mathrm{D}_{2}\right)}{f_{\mathrm{tr}}\left(\mathrm{n}-\mathrm{D}_{2}\right)} \\
& +\left(1+3 \frac{f_{\text {int }}\left(0-\mathrm{D}_{2}\right)-f_{\text {int }}\left(\mathrm{n}-\mathrm{D}_{2}\right)}{f_{\text {int }}\left(\mathrm{n}-\mathrm{D}_{2}\right)}\right) f_{\text {int }}\left(\mathrm{n}-\mathrm{D}_{2}\right) a(T) .
\end{aligned}
$$


Durch Vergleich von (8) mit (9) folgt:

$$
\begin{gathered}
f_{\mathrm{tr}}\left(\mathrm{o}-\mathrm{D}_{2}\right) / f_{\mathrm{tr}}\left(\mathrm{n}-\mathrm{D}_{2}\right)=1,0002, \\
\left(1+3^{\left.\frac{f_{\mathrm{int}}\left(\mathrm{o}-\mathrm{D}_{2}\right)-f_{\text {int }}\left(\mathrm{n}-\mathrm{D}_{2}\right)}{f_{\text {int }}\left(\mathrm{n}-\mathrm{D}_{2}\right)}\right)} f_{\text {int }}\left(\mathrm{n}-\mathrm{D}_{2}\right)=2,5\right.
\end{gathered}
$$

Die Messungen zeigen also, da $f_{\text {tr }}\left(o-D_{2}\right)$ um etwa $0,2 \%$ größer ist als $f_{\operatorname{tr}}\left(n-D_{2}\right)$. Dieser Unterschied kann zwar beim heutigen Stand der Theorie nicht erklärt werden, er ist aber auch nicht weiter überraschend, besitzt doch z. B. Para-Deuterium durch Zentrifugalaufweitung im Grundzustand der Rotation $(l=1)$ ein um etwa $1 \%$ größeres Trägheitsmoment als Ortho-Deuterium $(l=0){ }^{9}$.

Bei den Messungen an leichtem Wasserstoff im Grundzustand der Rotation wurde $\frac{\Delta \lambda}{\lambda}=\frac{\Delta \eta}{\eta}$ gefunden ${ }^{1,2}$. Dabei konnten aber sowohl $\frac{\Delta \lambda}{2}$ als auch $\frac{\Delta \eta}{\eta}$ nur mit einer Genauigkeit von $\pm 3 \cdot 10^{-4}$ gemessen werden. Der beim Deuterium gefundene Effekt von einigen Zehntel Promille ging also beim leichten Wasserstoff möglicherweise in den Fehlergrenzen unter.

Zur Deutung von Gl. (11) bieten sich zwei Möglichkeiten an. Wenn man $f_{\text {int }}\left(o-D_{2}\right)=f_{\text {int }}\left(n-D_{2}\right)$ annimmt, folgt daraus $f_{\text {int }}=2,5$. Dies ist aber unwahrscheinlich, denn $f_{\text {int }}$ sollte etwa gleich $\varrho D / \eta$ sein, und das ist für Deuterium bei $20,4{ }^{\circ} \mathrm{K}$ in guter

\footnotetext{
9 Aus den Rechnungen von Chapman und Cowling ${ }^{10}$ mit dem Modell der rauhen Kugeln folgt, daß $f_{\text {tr }}$ mit zunehmendem Trägheitsmoment abnimmt. Die rauhen Kugeln zeigen also ein analoges Verhalten; aber dieses Modell stimmt, insbesondere da es stetige Anregbarkeit der Rotationen voraussetzt, nicht mit dem wirklichen Verhalten der Deuterium-Moleküle überein.
}

Näherung gleich eins. Wenn man dagegen $f_{\text {int }}\left(\mathrm{o}-\mathrm{D}_{2}\right)$ $\neq f_{\text {int }}\left(\mathrm{n}-\mathrm{D}_{2}\right) \approx 1$ annimmt, ergeben sich große Unterschiede für die beiden Faktoren (aus $f_{\text {int }}\left(n-D_{2}\right)=1$ folgt z. B. $\left.f_{\text {int }}\left(o-D_{2}\right)=1,5\right)$. Unterschiede in dieser Größe würde man nicht erwarten. Beide Deutungen des Befundes (11) sind also unbefriedigend. Der Erwartung $f_{\text {int }} \approx 1$ und der Ähnlichkeit der beiden Deuterium-Modifikationen entsprechend sollte vielmehr der Faktor bei $a(T)$ in Gl. (9) etwa gleich eins sein. Die dritte Kurve in Abb. $1\left(4,3 \cdot 10^{-4}+a(T)\right)$ ist ein Kompromiß zwischen dieser Erwartung und den Meßergebnissen. Man sieht, daß die Kurve die Meßwerte schlecht wiedergibt. Sie geht gerade durch die äußeren Enden der Fehlerbalken.

Wenn also auch bezüglich $f_{\text {int }}$ noch keine volle Klärung erreicht worden ist, so kann doch als gesichertes Ergebnis der Arbeit angesehen werden, daß $f_{\text {tr }}$ für Ortho-Deuterium um einige Zehntel Promille größer ist als für Normal-Deuterium.

Die vorbildliche Zusammenarbeit beider Institute wurde nicht zuletzt durch die Gastfreundschaft von Herrn Prof. Dr. H. Witre, Darmstadt, ermöglicht. Der für die Versuche benötigte flüssige Wasserstoff entstammt einer Verflüssigungsanlage, die als Leihgabe der Deutschen Forschungsgemeinschaft dem EduardZintl-Institut zur Verfügung steht.

10 S. Chapman u. T. G. Cowling, The Mathematical Theory of Non-Uniform Gases, Cambridge University Press 1952. 